\title{
Rendimiento de 10 clones de camote (Ipomoea batatas L.) en Trujillo, La Molina, San Ramón y Huaral
}

Yield of ten sweetpotato (Ipomoea batatas L.) clones in Trujillo, La Molina, San Ramon and Huaral

Rember pinedo taco ${ }^{1}$, Gilberto rodríguez soto ${ }^{1}$ y Norma VALVERde Reyes ${ }^{1}$

\section{RESUMEN}

Evalúa diez avanzados clones de camote de pulpa naranja en las localidades de San Ramón, Huaral, Lima y Trujillo. El experimento de campo se llevó a cabo bajo un diseño de bloques completos al azar con dos bloques en las cuatro localidades. Las variables en estudio fueron: rendimiento total de las raíces reservantes, rendimiento comercial de raíces reservantes, rendimiento de follaje fresco y materia seca de raíces reservantes. En rendimiento de follaje fresco, Huambachero resultó el de mayor rendimiento, pero estadísticamente similar a los demás clones PZ08.153, PJ05.212, Jonathan, PZ06.029 y PJ05.052; en rendimiento comercial de raíces reservantes, los clones PJ05.212 (96.34 t.ha $\left.{ }^{-1}\right)$; PJ05.052 (85.13 t.ha $\left.{ }^{-1}\right)$; PH06.011 (39.59 t.ha ${ }^{-1}$ ) y PJ07.119 (37.77 t.ha $\left.{ }^{-1}\right)$ produjeron los rendimientos más altos en Trujillo, La Molina, San Ramón y Huaral, respectivamente. Respecto a los rendimientos totales de raíces reservantes, los mejores clones fueron PJ05.052 (116.37 t.ha ${ }^{-1}$ ); PJ05.052 (130.68 t.ha' ${ }^{-1}$ ); PJ07.691 (54.59 t.ha ${ }^{-1}$ y PZ08.153 (44.51 t.ha ${ }^{-1}$ ) para las localidades de Trujillo, La Molina, San Ramón y Huaral, respectivamente. Los rendimientos más altos en materia seca de raíces reservantes por hectárea fueron producidos por Huambachero (37.74 t.ha ${ }^{-1}$ en Trujillo y 12.16 t.ha ${ }^{-1}$ en Huaral); PJ07.691 (13.68 t.ha $\left.{ }^{-1}\right)$ en San Ramón, y PJ05.052 (18.76 t.ha ${ }^{-1}$ ) en La Molina.

Palabras clave: clones; Ipomoea batatas L.; localidad; rendimiento; raíces reservantes.

\begin{abstract}
Evaluate ten advanced orange flesh sweetpotato clones in the locations of San Ramón, Huaral, Lima y Trujillo. The field experiment was conducted under a randomized complete block design with two blocks across the four locations;
\end{abstract}

1 Universidad Nacional Agraria La Molina. Lima, Perú. 
total storage root yield; commercial storage root yield; fresh foliage yield and dry matter of storage root were evaluated. Huambachero showed the highest foliage yield but was statistically similar to PZ08.153, PJ05.212, Jonathan, PZ06.029 y PJ05.052; In terms of comercial storage root yield, PJ05.212 (96.34 t.ha ${ }^{-1}$ ); PJ05.052 (85.13 t.ha (1) $^{-1}$ PH06.011 (39.59 t.ha (1) y PJ07.119 (37.77 t.ha $^{-}$ 1) clones produced the highest yields at Trujillo, La Molina, San Ramon and Huaral, respectively. With regard to total storage root yield, the best clones were PJ05.052 (116.57 t.ha' (1) $^{-1}$ PJ05.052 (130.68 t.ha ${ }^{-1}$ ); PJ07.691 (54.59 t.ha (1) $^{-1}$ and PZ08.153 (44.51 t.ha ${ }^{-1}$ ) for Trujillo, La Molina, San Ramon and Huaral environments, respectively. The highest storage root dry matter yield per hectare were produced by Huambachero (37.34 t.ha ${ }^{-1}$ at Trujillo and 12.16 t.ha $a^{-1}$ at Huaral); PJ07.691 (13.68 t.ha ${ }^{-1}$ ) at San Ramon, and PJ05.052 (18.76 t.ha ${ }^{-1}$ ) at La Molina.

Keywords: clones; Ipomoea batatas L.; locality; yield; storage roots.

\section{INTRODUCCIÓN}

Los estudios de Interacción Genotipo x Ambiente (IGA) tienen por finalidad identificar cultivares con altos rendimientos para una región dada (Yan et al., 2001) e identificar condiciones ideales (Carneiro, 1998). Entre las técnicas estadísticas multivariadas se encuentra el AMMI (Efectos Principales Aditivos e Interacciones Multiplicativas) (Gauch, 2006). La estabilidad permite al genotipo ajustar su capacidad productiva a la más amplia variación ambiental (ChuangSheng et al., 1986). Los métodos multivariados son los más adecuados para evaluar la estabilidad porque permiten describir e interpretar los efectos de la interacción $\mathrm{G} \times$ A (Gauch y Furnas, 1991). Para explicar el efecto Interacción Genotipo por Ambiente (IGA) sobre el comportamiento de los clones se utiliza el análisis de estabilidad fenotípica (Furtado et al., 2006). La IGA es importante ya que la estabilidad de rendimiento de un clon en particular depende de su respuesta a diversos factores adversos, en etapas críticas del desarrollo de la planta (Crossa, Wescott y Gonzales et al., 1988; Márquez, 1991). La IGA es el factor que más interfiere en la identificación de los clones específicos para ambientes específicos, lo cual limita la precisión en la estimación de rendimiento (Snedecor y Cochran, 1980). Eberhart y Russell (1966), llegaron a la conclusión que una variedad estable es aquella que no interacciona con la localidad sino que responde mejor a los cambios ambientales. Por su parte, Hanson (1970), indica que un material estable es aquel que muestra la menor variación del rendimiento sobre todas las localidades de evaluación. Con respecto al valor nutricional del camote, el follaje contiene carbohidratos, proteínas y celulosa; y las raíces poseen alto contenido de calcio, fósforo, carbohidratos, entre otros elementos (Alvarado et al., 2009), el valor nutricional del follaje es muy parecido al de la raíz (Linares et al., 2008). En un estudio realizado sobre la absorción de nutrientes y distribución de la materia fresca y seca entre órganos de camote encontraron que la mayor producción de raíces comerciales es aproximadamente 36.5 t.ha ${ }^{-1}$ (Echer et al., (2009). 


\section{MATERIALES Y MÉTODOS}

La investigación se realizó en las localidades de San Ramón, Huaral, Lima y Trujillo, durante el año 2012 (tabla 1). La temperatura máxima se mantuvo entre $21.06^{\circ} \mathrm{C}$ y 30.8 y la mínima de 15.8 y $17.05^{\circ} \mathrm{C}$, en las localidades de Huaral y San Ramón.

Tabla 1. Coordenadas y rango longitudinal de las cuatro localidades

\begin{tabular}{ccccc}
\hline \multirow{2}{*}{ Localidades } & \multicolumn{2}{c}{ Coordenadas } & \multicolumn{2}{c}{ Rango Altitudinal } \\
& Latitud & Longitud & msnm & Región \\
\hline San Ramón & $11^{\circ} 08^{\prime} 25$ & $75^{\circ} 20^{\prime} 00$ & 850 & Junín \\
Huaral & $11^{\circ} 31^{\prime} 16^{\prime \prime}$ & $77^{\circ} 14^{\prime} 8^{\prime \prime}$ & 181 & Lima \\
Trujillo & $8^{\circ} 6^{\prime} 43^{\prime \prime}$ & $78^{\circ} 59^{\prime} 6^{\prime \prime}$ & 31.16 & Libertad \\
La Molina & $12^{\circ} 05^{\prime} 06$ & $76^{\circ} 57^{\prime} 00^{\prime \prime}$ & 238 & Lima \\
\hline
\end{tabular}

Fuente: SENAMHI, 2012

Se evaluaron ocho clones experimentales de camote y las comerciales Huambachero y Jonathan como testigos en cada repetición. Las variables estudiadas fueron: rendimiento de follaje fresco $\left(\right.$ t.ha $\left.^{-1}\right)$, rendimiento comercial de raíces reservantes $\left(\mathrm{t} . \mathrm{ha}^{-1}\right)$, rendimiento total de raíces reservantes $\left(\mathrm{t} \cdot \mathrm{ha}^{-1}\right)$ y rendimiento de materia seca de raíces reservantes $\left(\right.$ t.ha $\left.{ }^{-1}\right)$. Se desarrollaron modelos estadísticos empleando los programas "MINITAB 16", "SAS versión 8.0" y "R versión 3.1". Obtenidas las ecuaciones y teniendo los procesos claramente establecidos se procedió a analizar a los clones de mayor rendimiento, estables y la interacción genotipo por ambiente ( $\mathrm{G} \times \mathrm{A}$ ) en este caso se utilizó el término de clones por localidad $(\mathrm{C} \times \mathrm{L})$ en cuatro localidades de estudio.

\section{RESULTADOS Y DISCUSIÓN}

En la tabla 2, se muestra el análisis de varianza combinado. El rendimiento de follaje fresco, mostró diferencias estadísticas altamente significativas para localidad y clon, mientras que para bloques/localidad e interacción clon x localidad $(\mathrm{C} \times \mathrm{L})$ no existen diferencias significativas, por lo que no fue necesario realizar evaluaciones por localidades. El rendimiento comercial de raíces reservantes, presentó diferencias estadísticas altamente significativas para localidad, bloques/localidad y para la interacción C x L, mientras que para los clones se observó diferencias significativas estadísticamente. En cuanto a rendimiento total de raíces reservantes, existe diferencia estadística altamente significativa para localidad, bloque/localidad y para interacción C x L, pero no diferencias significativas para clones. En rendimiento de materia seca de raíces reservantes, existen diferencias altamente significativas para localidad e interacción C x L, bloques/localidad y clones resultó ser no significativo. Para rendimiento RCRR, RT y RMS la interacción C x L indica que los clones no tuvieron un comportamiento igual en las localidades, por lo cual es necesario realizar las evaluaciones por localidades. 
Tabla 2. Análisis varianza combinado para rendimiento: follaje fresco (t.ha $\left.{ }^{-1}\right)$

\begin{tabular}{|c|c|c|c|c|c|}
\hline \multirow{2}{*}{ Fuente de variación } & \multirow{2}{*}{ GL } & \multicolumn{4}{|c|}{ CUADRADOS MEDIOS } \\
\hline & & RFF & RCRR & RT & RMS \\
\hline Localidad (L) & 3 & $0.15 * *$ & $4312.21 * *$ & $6428.92 * *$ & $516.06 * *$ \\
\hline Bloques/Localidad & 4 & 0.003 & $211.33 * *$ & $131.41 * *$ & 3.33 \\
\hline Clon $(\mathrm{C})$ & 9 & $0.01 * *$ & $1187.94 *$ & 1790.96 & 85.96 \\
\hline $\mathrm{C} \times \mathrm{L}$ & 27 & 0.002 & $463.76 * *$ & $808.99 * *$ & $60.57 * *$ \\
\hline Error conjunto & 36 & 0.002 & 14.4 & 24.29 & 4.98 \\
\hline Total & 79 & & & & \\
\hline
\end{tabular}

$\mathrm{RFF}=$ Rendimiento Follaje Fresco, RCRR = Rendimiento Comercial Raíces Reservantes, $\mathrm{RT}=$ Rendimiento Total, RMS = Rendimiento Materia Seca.

En la prueba de comparación de medias en promedio de localidades (tabla 3), la variedad Huambachero presentó mayor respuesta con 72.48 t.ha ${ }^{-1}$, estadísticamente similar a todos los demás clones; pero diferentes a los clones PH06.011 (27.33 t.ha' 1), PJ07.691 (19.84 t.ha-1) y PJ07.119 (18.45 t.ha (1) $^{-1}$; el clon PJ07.119 se ubicó en el último lugar, estadísticamente similar a todos los demás clones, excepto a la variedad Huambachero y al clon PZ08.153. La comparación de medias para las cuatro localidades en promedio de clones, Huaral, Trujillo y La Molina, mostraron mayor respuesta y son estadísticamente similares; pero diferentes a San Ramón. Velarde y Gómez (2003), indican rendimientos para follaje fresco en un rango de 14.4 a 27.12 t.ha ${ }^{-1}$, y Quispe (2005), 26.66 a 16.24 t.ha ${ }^{-1}$ ambos difieren con los resultados del estudio.

Tabla 3. Comparación de medias, prueba de Tukey, rendimiento follaje fresco (t.ha $\left.{ }^{-1}\right)$ en promedio de localidades y rendimiento follaje fresco $\left(\mathrm{t}\right.$ h $\left.\mathrm{a}^{-1}\right)$ en promedio de clones.

\begin{tabular}{|c|c|c|c|c|c|c|c|c|}
\hline \multicolumn{6}{|c|}{ Rendimiento follaje fresco $\left(\mathrm{t} \mathrm{ha}^{-1}\right)$ en promedio de localidades. } & \multicolumn{3}{|c|}{$\begin{array}{l}\text { Rendimiento follaje fresco }\left(\mathrm{t} \cdot \mathrm{ha}^{-1}\right) \\
\text { en promedio de clones. }\end{array}$} \\
\hline Clones & Medias & $\begin{array}{l}\text { Significa- } \\
\text { ción }\end{array}$ & Clones & Medias & $\begin{array}{l}\text { Significa- } \\
\text { ción }\end{array}$ & Clones & Medias & $\begin{array}{l}\text { Significa- } \\
\text { ción }\end{array}$ \\
\hline Huambachero & 72.48 & $\mathrm{a}$ & PJ05.052 & 32.94 & $\mathrm{a} b$ & Huaral & 51.3 & $\mathrm{a}$ \\
\hline PZ08.153 & 58.47 & $\mathrm{a}$ & PH07.058 & 28.43 & $\mathrm{ab}$ & Trujillo & 50.12 & $\mathrm{a}$ \\
\hline PJ05.212 & 39.24 & $a b$ & РH06.011 & 27.33 & $\mathrm{~b}$ & La Molina & 37.47 & $\mathrm{a}$ \\
\hline Jonathan & 37.29 & $\mathrm{a} b$ & PJ07.691 & 19.84 & $\mathrm{~b}$ & San Ramón & 9.98 & $\mathrm{~b}$ \\
\hline PZ06.029 & 34.74 & $\mathrm{ab}$ & PJ07.119 & 18.45 & $\mathrm{~b}$ & & & \\
\hline
\end{tabular}

La tabla 4, muestra los resultados alcanzados en las cuatro localidades para rendimiento comercial (t.ha ${ }^{-1}$ ) de raíces reservantes. En la localidad de Huaral (H), el clon PJ07.119 se muestra en el primer lugar con 37.78 t.ha ${ }^{-1}$, similar estadísticamente a todos los demás clones; excepto al clon PJ07.691 con 25.99, que ocupa el último lugar, similar estadísticamente a todos los demás clones pero diferente al clon PJ07.119. 
Estos resultados son menores a los obtenidos por Molina y Aybar (2010) con rendimientos promedios de 15.65 a 25.46 t.ha $^{-1}$.

En la localidad de La Molina el clon PJ05.052 (85.14 t.ha $\left.{ }^{-1}\right)$ obtuvo el mayor rendimiento y, resultó similar estadísticamente a los clones PJ05.212 (76.12 t.ha-1) y PH06.011 (72.54 t.ha $\left.{ }^{-1}\right)$; mientras que el resto de los clones tuvieron rendimientos inferiores, ocupando el último lugar la variedad Huambachero con 19.86 t.ha $^{-1}$, similar estadísticamente a la variedad Jonathan y los clones PJ07.691, PJ07.119 y PZ06.029 con 23.13, 25.11, 43.55 y 44.05 t.ha ${ }^{-1}$. Reynoso (2003), afirma que 10.6 t.ha ${ }^{-1}$ es el rendimiento de la variedad Jonathan; resultados superiores fueron encontrados en la presente investigación con respecto a la variedad Jonathan. En localidad San Ramón (SR): el clon con mayor rendimiento fue PH06.011 con un rendimiento de 39.59 t.ha ${ }^{-1}$ y es similar estadísticamente al clon PJ07.691 (36.11 t.ha ${ }^{-1}$ ); los demás clones presentaron rendimientos menores, siendo la variedad Huambachero el de menor rendimiento con 9.08 t.ha ${ }^{-1}$. Para esta localidad (San Ramón), Echer et al. (2009) encontró que la mayor producción de raíces comerciales es 36.5 t.ha ${ }^{-1}$.

Tabla 4: Comparación de medias mediante la prueba de Tukey al 0.05 de probabilidad para rendimiento comercial $\left(\mathrm{t}_{\mathrm{h}} \mathrm{h}^{-1}\right)$ de raíces reservantes

\begin{tabular}{|c|c|c|c|c|c|}
\hline \multicolumn{3}{|c|}{ Huaral } & \multicolumn{3}{|c|}{ La Molina } \\
\hline clon & media & sign & clon & media & sign \\
\hline PJ07.119 & 37.78 & a & PJ05.052 & 85.14 & a \\
\hline PH06.011 & 37.29 & $a b$ & PJ05.212 & 76.12 & $a b$ \\
\hline PJ05.212 & 35.94 & $a b$ & PH06.011 & 72.54 & $\mathrm{a} \mathrm{b} \mathrm{c}$ \\
\hline PZ08.153 & 35.8 & $a b$ & PZ08.153 & 57.66 & b c d \\
\hline Huambachero & 35.8 & $a b$ & PH07.058 & 47.29 & $\mathrm{~cd} \mathrm{e}$ \\
\hline Jhonathan & 29.55 & $a b$ & PZ06.029 & 44.05 & $d$ e $f$ \\
\hline PH07.058 & 29.38 & $a b$ & PJ07.119 & 43.55 & $d$ e $f$ \\
\hline PJ05.052 & 28.86 & $a b$ & PJ07.691 & 25.11 & ef \\
\hline PZ06.029 & 27.9 & $a b$ & Jhonathan & 23.13 & ef \\
\hline PJ07.691 & 25.99 & $\mathrm{ab}$ & Huambachero & 19.86 & $\mathrm{f}$ \\
\hline \multicolumn{3}{|c|}{ San Ramón } & \multicolumn{3}{|c|}{ Trujillo } \\
\hline clon & media & sign & clon & media & sign \\
\hline PH06.011 & 39.59 & $\mathrm{a}$ & PJ05.212 & 96.34 & $\mathrm{a}$ \\
\hline PJ07.691 & 36.11 & $\mathrm{ab}$ & PJ05.052 & 91.6 & a \\
\hline PZ06.029 & 34.32 & $\mathrm{~b} \mathrm{c}$ & Huambachero & 80.88 & a \\
\hline PJ05.212 & 34.15 & $\mathrm{bc}$ & PH06.011 & 79.33 & $\mathrm{~b}$ \\
\hline PZ08.153 & 31.26 & $\mathrm{~cd}$ & PZ06.029 & 66.91 & $\mathrm{~b}$ \\
\hline PH07.058 & 30.86 & $\mathrm{~cd}$ & PZ08.153 & 48.58 & $\mathrm{c}$ \\
\hline PJ07.119 & 29.53 & $\mathrm{~d} \mathrm{e}$ & PH07.058 & 47.96 & $\mathrm{~d}$ \\
\hline PJ05.052 & 28.19 & $\mathrm{de}$ & PJ07.119 & 47.5 & $\mathrm{~d}$ \\
\hline Jhonathan & 26.19 & $\mathrm{e}$ & PJ07.691 & 31.7 & e \\
\hline Huambachero & 9.08 & $\mathrm{f}$ & Jhonathan & 31.7 & $\mathrm{f}$ \\
\hline
\end{tabular}


En la localidad Trujillo (T), el clon PJ05.212 (96.340 t.ha-1) se ubicó en el primer lugar y es similar estadísticamente al clon PJ05.052 (91.60 t.ha $\left.{ }^{-1}\right)$; la variedad Jonathan con 19.72 t.ha $^{-1}$, se encuentra en el último lugar y es diferente a los demás clones. En un estudio realizado sobre validación de camote forrajero se encontró rendimientos de follaje que varían de 27.20 a 98.79 t.ha ${ }^{-1}$ (Quispe, 2005)

En la figura 1, al realizar el análisis AMMI, se detalla la representación gráfica del biplot, las líneas punteadas de color rojo representa a los sectores de las localidades y los triángulos de color azul representa a los clones. Los clones situados cerca del centro de la gráfica fueron menos sensibles al cambio del ambiente. Los clones PZ06.029 (39.5 t.ha ${ }^{-1}$ ) y PJ07.119 (43.29 t.ha ${ }^{-1}$ ) fueron los más estables en la respuesta del rendimiento de raíces comerciales cuando fueron probados en las cuatro localidades. Las variedades y clones con mejor adaptación a localidades resultaron Huambachero, PJ05.052, PZ08.153 y Jonathan para Trujillo, La Molina, San Ramón y Huaral respectivamente; es decir, resultaron ser los que más aportaron a la interacción clon x localidad, debido a que estuvieron más alejados del centro de biplot. Esto demuestra que los clones estudiados presentaron un comportamiento no uniforme a traves del conjunto de localidades en que fueron probados.

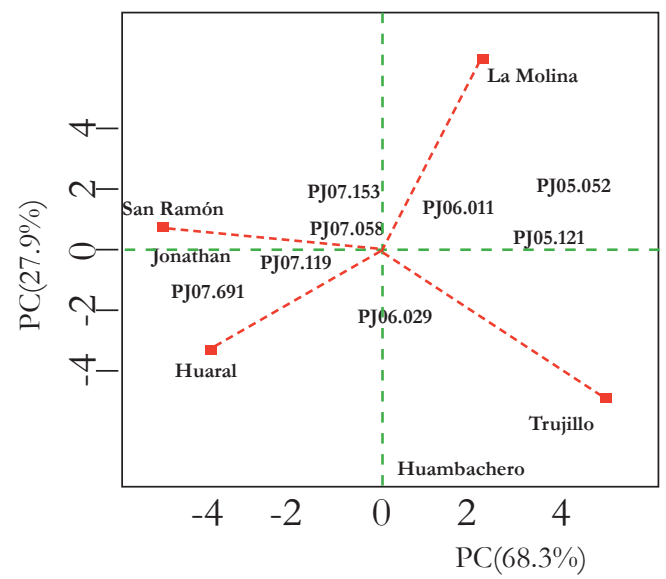

Figura 1. Representación de C x L respecto a los dos primeros ejes de componentes principales del análisis AMMI para rendimiento comercial de raíces reservantes ( $\mathrm{t}$.ha $\mathrm{a}^{-1}$ ) de clones evaluados en cuatro localidades

Al comparar las medias de los clones (tabla 4), para rendimiento total (t.ha $\left.{ }^{-1}\right)$ de raíces reservantes en la localidad Huaral $(\mathrm{H})$, el clon PZ08.153 con 44.510 t.ha ${ }^{-1}$ alcanzó el primer lugar y es diferente de los demás clones; mientras que el clon PJ07.691 el último lugar con 29.26 t.ha $^{-1}$ y es similar a los demás clones, excepto el clon PZ08.153. Reynoso (2003), para la variedad Jonathan halló rendimientos desde 13.8 hasta 25.5 t.ha ${ }^{-1}$, menor a lo hallado en el presente estudio. En la localidad de La Molina, con el clon PJ05.052 se halló el mejor rendimiento de 130.68 t.ha ${ }^{-1}$, ocupando el último lugar la variedad Huambachero con 25.62 t.ha $^{-1}$ y resultó similar estadísticamente a la variedad Jonathan y los clones PJ07.691 y PJ07.119 con 27.25, 27.95 y 46.94 t.ha ${ }^{-1}$. 
Estos resultados coinciden con Reynoso (2003) quien afirma que la variedad Huambachero alcanza rendimiento de hasta 32.1 t.ha ${ }^{-1}$. En la localidad San Ramón (SR), el clon PJ07.691 resultó con 54.59 t.ha ${ }^{-1} \mathrm{y}$ es estadísticamente diferente a los demás clones, la variedad Huambachero con 13.55 t.ha-1 se relegó en el último lugar y es diferente de los demás clones (tabla 8). Mientras que en la localidad Trujillo (T), el clon PJ05.052 (116.57 t.ha ${ }^{-1}$ ) obtuvo el mejor rendimiento y es similar estadísticamente a los clones PH06.011 (103.45 t.ha $\left.{ }^{-1}\right)$ y PJ05.212 (102.29 t.ha $\left.\mathrm{A}^{-1}\right)$; pero diferente a los demás clones, la variedad Jonathan con 23.02 t.ha ${ }^{-1}$ se ubicó en el último lugar y es similar estadísticamente al clon PJ07.691. Estos bajos rendimientos podrían ser por el tipo de suelo, lo cual puede haber afectado negativamente al crecimiento del cultivo. Además en anteriores trabajos de Gong et al. (1990) demostraron que la sequía y el estrés causado por otros factores ambientales adversos cuya duración sea mayor de 20 días en cualquier etapa del periodo de vegetativo, disminuye el rendimiento de raíces de camote.

Tabla 5: Comparación de medias mediante la prueba de Tukey al 0.05 de probabilidad para rendimiento total $\left(\mathrm{t} \cdot \mathrm{ha}^{-1}\right)$ de raíces reservantes.

\begin{tabular}{|c|c|c|c|c|c|}
\hline \multicolumn{3}{|c|}{ Huaral } & \multicolumn{3}{|c|}{ La Molina } \\
\hline clon & media & sign & clon & media & sign \\
\hline PZ08.153 & 44.51 & $\mathrm{a}$ & PJ05.052 & 130.68 & $\mathrm{a}$ \\
\hline PJ07.119 & 40.73 & $\mathrm{~b}$ & PH06.011 & 80.94 & $\mathrm{~b}$ \\
\hline PJ05.212 & 38.61 & $\mathrm{~b}$ & PJ05.212 & 80.75 & $\mathrm{~b}$ \\
\hline PH06.011 & 38.46 & $\mathrm{~b}$ & PZ08.153 & 65.11 & $\mathrm{bc}$ \\
\hline Huambachero & 38.19 & $\mathrm{~b}$ & PH07.058 & 59.53 & $\mathrm{bc}$ \\
\hline Jhonathan & 34.78 & $\mathrm{~b}$ & PZ06.029 & 54.32 & $\mathrm{bcd}$ \\
\hline PJ05.052 & 33.8 & $\mathrm{~b}$ & PJ07.119 & 46.94 & de \\
\hline PZ06.029 & 31.88 & $\mathrm{~B}$ & PJ07.691 & 27.95 & $\mathrm{de}$ \\
\hline PH07.058 & 31.3 & $\mathrm{~B}$ & Huambachero & 27.25 & $\mathrm{de}$ \\
\hline PJ07.691 & 29.26 & $\mathrm{~b}$ & Jhonathan & 25.62 & $\mathrm{e}$ \\
\hline \multicolumn{3}{|c|}{ San Ramón } & \multicolumn{3}{|c|}{ Trujillo } \\
\hline clon & Media & sign & clon & Media & sign \\
\hline PJ07.691 & 54.59 & $\mathrm{a}$ & PJ05.052 & 116.57 & $\mathrm{a}$ \\
\hline PH06.011 & 43.56 & $\mathrm{~b}$ & PH06.011 & 103.45 & $a b$ \\
\hline PH07.058 & 38.23 & bc & PJ05.212 & 102.29 & $\mathrm{ab}$ \\
\hline PZ06.029 & 37.89 & $\mathrm{c}$ & Huambachero & 89.12 & $\mathrm{bc}$ \\
\hline PJ05.212 & 37.33 & c & PZ06.029 & 71.37 & $\mathrm{~cd}$ \\
\hline PJ07.119 & 36.08 & $\mathrm{~cd}$ & PZ08.153 & 60.07 & de \\
\hline PZ08.153 & 34.08 & cde & PH07.058 & 59.71 & de \\
\hline PJ05.052 & 31.07 & de & PJ07.119 & 52.86 & de \\
\hline Huambachero & 28.13 & $\mathrm{e}$ & PJ07.691 & 37.55 & ef \\
\hline Jhonathan & 13.55 & $\mathrm{f}$ & Jhonathan & 23.03 & $\mathrm{f}$ \\
\hline
\end{tabular}


En el Análisis AMMI (figura 2), los clones próximos al centro de la gráfica de doble representación fueron los que menos contribuyeron a la interacción clon x localidad. Los clones PZ06.029 (48.86 t.ha ${ }^{-1}$ ) y PJ07.119 (44.15 t.ha- ${ }^{1}$ ) fueron los más estables en las cuatro localidades. La agrupación de los clones y localidades en un solo cuadrante señala una asociación positiva entre ellos. Los clones más alejados del centro del biplot indican una mejor adaptación, por ejemplo la variedad Huambachero y los clones PJ05.052 y PJ07.691, se adaptaron mejor en Trujillo, La Molina y en San Ramón. Los principales efectos del medio ambiente fueron altamente significativos, lo que implica que la diferencia de respuestas genotípicas a ambientes estaba relacionada con los factores propios de cada localidad tales como condiciones de humedad, temperatura, radiación solar, fertilidad del suelo del suelo durante el crecimiento del material genético en evaluación (Moussa et al., 2011).

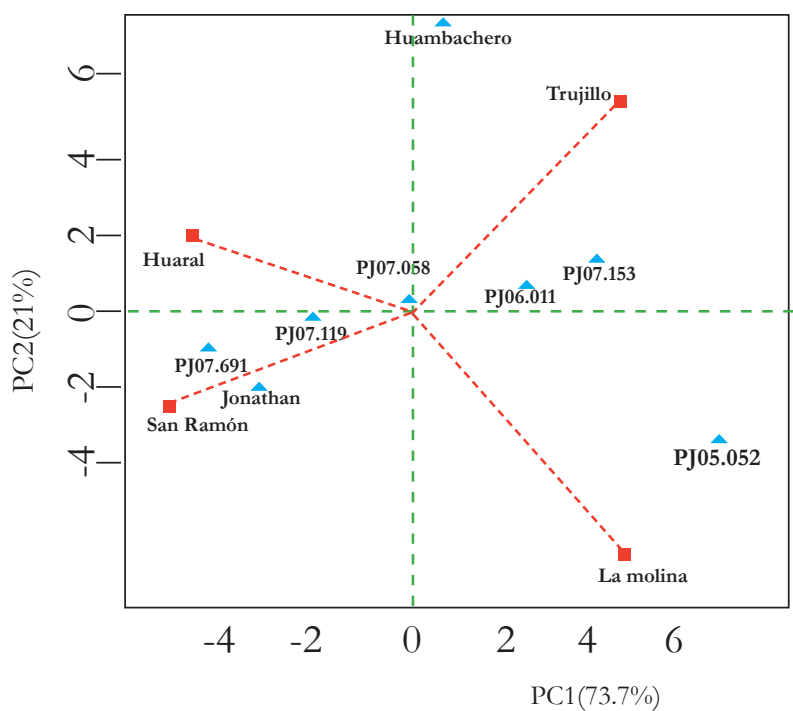

Figura 2. Representación de C x L, respecto a los dos primeros ejes de componentes principales del análisis AMMI para rendimiento total de raíces reservantes $\left(\right.$ t.h $\left.^{-1}\right)$ de clones evaluados en cuatro localidades.

$\mathrm{Al}$ realizar la comparación de medias mediante Tukey al 0.05 de probabilidad para rendimiento de materia seca en la tabla 6, para la localidad Huaral (LH), la variedad Huambachero muestra el rendimiento con 12.16 t.ha ${ }^{-1}$ y el clon PH06.011 el último lugar y son similares estadísticamente a todos los demás clones. En la localidad La Molina (LM) el clon PJ05.052 alcanzó el mayor rendimiento con 18.76 tha $^{-1} \mathrm{y}$ resultó similar estadísticamente a los clones PJ05.212, PZ08.153, PH07.058, PH06.011, PZ06.029 y PJ07.119; el clon PJ07.691 con 6.04 t.ha $^{-1}$ es el de menor rendimiento y es similar estadísticamente a todos los 
demás clones, pero diferente de los clones PJ05.052, PJ05.212 y PZ08.153. En la localidad San Ramón (SR), con la variedad PJ07.691 se halló el mejor rendimiento con 13.68 t.ha $^{-1}$; a su vez la variedad Huambachero ocupó el último lugar y son similares estadísticamente a todos los demás clones. En la localidad Trujillo (T), la variedad Huambachero resultó con 37.34 t.ha ${ }^{-1} \mathrm{y}$ es similar estadísticamente al clon PJ05.052, pero diferente a los demás clones; la variedad Jonathan con 6.62 t.ha ${ }^{-1}$ por tener el menor rendimiento, ocupó el último lugar y es similar estadísticamente a los clones PH07.058, PJ07.119 y PJ07.691.

Tabla 6. Comparación de medias Tukey al 0.05 de probabilidad para rendimiento de materia seca $\left(\right.$ t.ha $\left.^{-1}\right)$ de raíces reservantes

\begin{tabular}{|c|c|c|c|c|c|}
\hline \multicolumn{3}{|c|}{ Huaral } & \multicolumn{3}{|c|}{ La Molina } \\
\hline clon & Media & sign & clon & Media & sign \\
\hline Huambachero & 12.16 & $\mathrm{a}$ & PJ05.052 & 18.76 & a \\
\hline PZ08.153 & 11.68 & $\mathrm{a}$ & PH06.011 & 17.24 & $a b$ \\
\hline PJ07.119 & 11.05 & a & PJ05.212 & 17.17 & $a b$ \\
\hline PJ05.052 & 9.93 & $\mathrm{a}$ & Huambachero & 14.99 & $a b c$ \\
\hline PJ05.212 & 9.24 & $\mathrm{a}$ & PZ06.029 & 13.95 & $a b c$ \\
\hline Jhonathan & 9.05 & $\mathrm{a}$ & PZ08.153 & 13.02 & $a b c$ \\
\hline PZ06.029 & 8.81 & $\mathrm{a}$ & РH07.058 & 9.32 & $a b c$ \\
\hline PH07.058 & 8.05 & $\mathrm{a}$ & PJ07.119 & 7.72 & $\mathrm{bc}$ \\
\hline PJ07.691 & 7.39 & $\mathrm{a}$ & PJ07.691 & 6.64 & $\mathrm{bc}$ \\
\hline РH06.011 & 6.66 & a & Jhonathan & 6.04 & c \\
\hline \multicolumn{3}{|c|}{ San Ramón } & \multicolumn{3}{|c|}{ Trujillo } \\
\hline clon & Media & sign & clon & Media & sign \\
\hline PJ07.691 & 12.16 & a & Huambachero & 18.76 & $\mathrm{a}$ \\
\hline PZ08.153 & 11.68 & $\mathrm{a}$ & PJ05.052 & 17.24 & $a b$ \\
\hline PH06.011 & 11.05 & a & PH06.011 & 17.17 & $\mathrm{bc}$ \\
\hline PJ05.212 & 9.93 & a & PJ05.212 & 14.99 & $\mathrm{~cd}$ \\
\hline PJ07.119 & 9.24 & a & PZ06.029 & 13.95 & cde \\
\hline PJ05.052 & 9.05 & $\mathrm{a}$ & PZ08.153 & 13.02 & cde \\
\hline PZ06.029 & 8.81 & $\mathrm{a}$ & PH07.058 & 9.32 & def \\
\hline Huambachero & 8.05 & $\mathrm{a}$ & PJ07.119 & 7.72 & ef \\
\hline PH07.058 & 7.39 & $\mathrm{a}$ & PJ07.691 & 6.64 & ef \\
\hline Jhonathan & 6.66 & a & Jhonathan & 6.04 & $\mathrm{f}$ \\
\hline
\end{tabular}

El biplot modelo AMMI en la interacción clon x localidad permite la visualización de las diferencias en la interacción (figura 1).de efectos principales (figura 
3). El clon PZ06.029, mostró estabilidad por estar cerca al centro del biplot. Los más inestables fueron en variedades Huambachero y Jonathan; mientras que en clones fueron PJ07.691, PH07.058 y PJ05.052. Los clones más alejados del centro del biplot indican una mejor adaptación, por ejemplo Huambachero en Trujillo, el clon PH07.058 en La Molina, la variedad Jonathan San Ramón y en Huaral el clon PJ07.691. En el estudio el primer y segundo eje clasifica a los clones entre grupos, al respecto Crossa et al. (1991), sostienen que los ensayos multilocales clasifica a los genotipos en tres grupos.

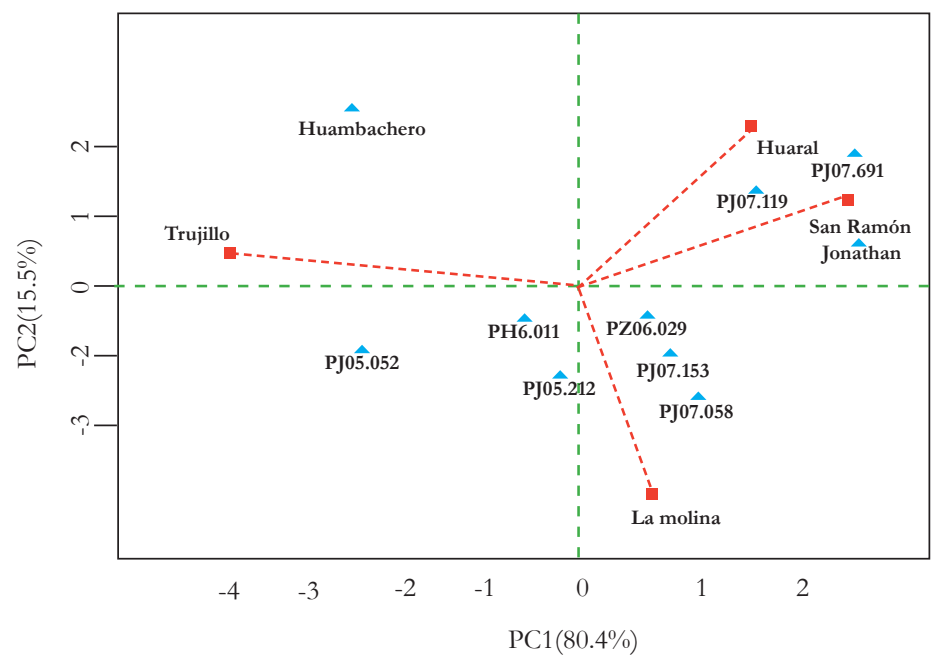

Figura 3. Representación de clones y localidades respecto a los dos primeros ejes de componentes principales del análisis AMMI de materia seca de raíces reservantes (t.ha ${ }^{-1}$ )

\section{CONCLUSIONES}

El clon PZ06.029 presentó estabilidad en las diferentes localidades para las variables rendimiento comercial, total y materia seca; mientras que el clon PJ07.119 solo presentó estabilidad en la variable raíces totales.

La variedad Huambachero resultó ser el de mayor rendimiento en t.ha ${ }^{-1}$ de follaje fresco, pero estadísticamente similar a los clones PZ08.153, PJ05.212, Jonathan, PZ06.029 y PJ05.052.

Para rendimiento comercial, en los clones PJ05.212 (96.34 t.ha ${ }^{-1}$ ); PJ05.052 (85.14 t.ha ${ }^{-1}$ ); PH06.011 (39.59 t.ha ${ }^{-1}$ ) y PJ07.119 (37.78 t.ha $\left.{ }^{-1}\right)$ se hallaron los rendimientos más altos en Trujillo, La Molina, San Ramón y Huaral, respectivamente.

Para rendimiento total de raíces reservantes, los mejores clones fueron PJ05.052

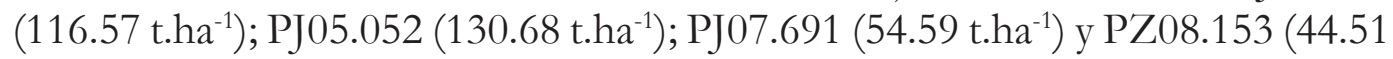
t.ha ${ }^{-1}$ ) para las localidades de Trujillo, La Molina, San Ramón y Huaral. 
Los rendimientos más altos para materia seca de raíces reservantes por hectárea fueron producidos por Huambachero (37.34 t.ha- en Trujillo y 12.16 t.ha ${ }^{-1}$ en Huaral); PJ07.691 (13.68 t.ha ${ }^{-1}$ ) en San Ramón y PJ05.052 (18.76 t.ha ${ }^{-1}$ ) en La Molina.

\section{REFERENCIAS BIBLIOGRÁFICAS}

Alvarado Flores, Karen Sofía y otros. 2009. Creación del Broker MKV para la exportación de camote y otros productos agrícolas no tradicionales a España. Guayaquil: Escuela Superior Politecnica del Litoral. Facultad de Economía y Negocios.

Carneiro, Pedro. 1998. Novas metodologías de analise da adaptabilidade e estabilidade de comportamiento. Dissertacão (Maestrado) - Universidad Federal de Vinosa.

Crossa J.; Wescott B. y Gonzales C. 1988. «Analysing yield stability of maize genotypes using a spatial model». Theor. Appl Genet. Vol. 75, N³: 863-868.

Crossa, J. y otros. 1991. «AMMI adjustment for statistical analysis of two international maize cultivar trials». Theoretical and Applied Genetic. Vol. 81. 27-37.

Eberhart and W. A. Rusell. 1966. «Stability parameters for comparing varieties». Crop sciences. Vol. 6, $\mathrm{N}^{\circ}$ 1: Crop Science Society of America. 36-40.

Echer, Fabio; Dominato, Julio y Creste, José. 2009. «Absorção de nutrientes e distibuição da massa fresca e seca entre órgãos de batata-doce». Horticultura Brasileira. Vol. 27. N². 167-182.

Furtado, Daniel y otros. 2006. «Statistical models in agriculture: Biometrical methods for evaluating phenotypic stability in plant breeding». Cerne, V.12. N4. Lavras: Universidade Federal de Lavras. 373-388

Gauch, Hugh y Furnas, Richard. 1991. «Statistical analysis of yield trails with MATMODEL». Agronomy Journal. Vol. 83 N 5. 916-920.

Gauch, Hugh. 2006. Statistical Analysis of Yield Trials by AMMI and GGE. Crop Sci. Vol. 46 No. 4. 1488-1500.

Gong, Y. y Wang G. 1990. «An investigation of the effect of drought stress on growwth of sweetpotato and measures to improve drought resistance and stabilize yields». Agricultural Sciences. N 1. Zhejiang Journal. 26-29.

Hanson, W. 1970. «Genotypic stability ». Theor. Appl. Genet. Vol. 40. 226-231.

Chuang-Sheng, Lin; Binns, Michael y Lefkovitch, Leonard. 1986. «Stability analysis: where do we stand?». Crop science. Vol. 26, N 5. 894-900. 
Linares, Edelmira; Bye, Robert; Rosa-Martínez, Daniel y Pereda, Rogelio. 2008. «El camote». Biodiversitas. Vol. 81. CONABIO. Universidad Nacional Autónoma de México. 11- 15.

Márquez, Fidel. 1991. Genotecnia vegetal, Métodos, teoría, resultados. Tomo III. México DF: AGT editor. S.A.

Molina, Oscar y Aybar, Juan. 2010. Desarrollo de variedades de camote para consumo fresco. Revista Institucional. Lima: AgroInnova. Año 1. Edición N² 2

Moussa, Sam; El-Aal, Haa y El-Fadl, Nia. 2011. «Stability study of sweet potato yield and its component carácter sunder different environments by joint regression analysis». Hort. Sci \& Ornamental plants . Vol. 3, N 1. 43-54.

Quispe, Cáceres. 2005. «Validación del nuevo cultivar del camote forrajero Lactogénico». Lima: Ciencia y Desarrollo. Vol. 6. 7-30.

Reynoso, Daniel. 2003. Desarrollo de productos de Camote para América Latina: Materia Seca de las Raices de Camote. Identificación de variedades para procesamiento. Informe Técnico final del Proyecto . Lima: FONTAGRO. CIP.

Snedecor, George y Cochran, Wiliam. 1980. Statistical methods. $7^{\text {th }}$ ed. A mes: Lowa State: University Press.

Velarde, León y Gómez, Carlos. 2003. Desarrollo de productos de Camote para América Latina: Camote Forrajero: Utilización de camote de doble propósito en la alimentación animal. Lima: CIP - UNALM .

Yan, Weikai y Hunt, La. 2001. «Interpretation of genotype x environment interaction for winter wheat yield in Ontario». Crop Science. Vol.41: 19-25.

Presentado: $24 / 01 / 2017$

Aceptado: 28/04/2017

\section{Correspondencia.}

Norma C. Valverde

rpinedo@lamolina.edu.pe 